\title{
Chromatin Dynamics of Circadian Transcription
}

\author{
Lorena Aguilar-Arnal • Paolo Sassone-Corsi
}

Published online: 6 February 2015

(C) Springer International Publishing AG 2015

\begin{abstract}
The molecular circadian clock orchestrates the daily cyclical expression of thousands of genes. Disruption of this transcriptional program leads to a variety of pathologies, including insomnia, depression, and metabolic disorders. Circadian rhythms in gene expression rely on specific chromatin transitions which are ultimately coordinated by the molecular clock. As a consequence, a highly plastic and dynamic circadian epigenome can be delineated across different tissues and cell types. Intriguingly, genome topology appears to coordinate cyclic transcription at circadian interactomes, in which circadian genes are in physical contact within the cell nucleus in a time-specific manner. Moreover, the clock machinery shows functional interplays with key metabolic regulators, thereby connecting the circadian epigenome to cellular metabolism. Unraveling the molecular aspects of such interplays is likely to reveal new therapeutic strategies towards the treatment of metabolic disorders.
\end{abstract}

Keywords Circadian rhythms · Chromatin · Epigenetics · Sirtuins $\cdot \mathrm{NAD}^{+}$

\section{Introduction}

Life has evolved to adapt to daily changes in the environment provided by the Earth's rotation. As a consequence, 24-h period rhythms in physiology and behavior are observed in almost all living organisms [1]. Indeed, circadian rhythms are remarkably pervasive across evolution and can be traced back

This article is part of the Topical Collection on Gene Regulation

L. Aguilar-Arnal $\cdot$ P. Sassone-Corsi $(\square)$

Center for Epigenetics and Metabolism, Unit 904 of INSERM,

Department of Biological Chemistry, University of California,

Irvine, CA 92697, USA

e-mail: psc@uci.edu to the ancient life form Archaebacteria [2••]. In mammals, circadian rhythms are apparent in sleep-wake cycles, feeding behavior, circulating levels of hormones (e.g., melatonin, insulin, leptin, adrenocorticotrophic hormone, or cortisol), body temperature, bowel movements, heartbeat, muscle performance, etc., all of which fluctuate with a period of $\sim 24 \mathrm{~h}$ [3]. These oscillations are sustained by the circadian clock, an endogenous timekeeper that provides the organisms with the ability to anticipate daily fluctuations in the environment and adapt accordingly. Thus, circadian rhythms persist in the absence of environmental cues to provide internal temporal organization and simultaneously, they can be entrained by certain external cues (zeitgebers) to ensure synchrony with the environment. Light is the most powerful zeitgeber [4].

The mammalian circadian system is organized as a hierarchy of oscillators, with the master pacemaker located in the suprachiasmatic nucleus (SCN) of the hypothalamus [5]. Circadian oscillators are present in almost all tissues of an organism, and the SCN orchestrates their coordinated function. In mammals, the $\mathrm{SCN}$ is composed of $\sim 20,000$ neurons whose activity is coupled and oscillate in synchrony. Coupling mechanisms involve precise network architecture and the release of specific neuropeptides and neurotransmitters, including neuropeptide Y (NPY), serotonin, vasoactive intestinal neuropeptide (VIP), and arginine vasopressin (AVP). The SCN directly receives photic information from the retina through the retinohypothalamic tract and feedback from certain target structures such as the pineal gland [6]. Through an intricate system of efferent projections and humoral signaling, the SCN entrains ancillary oscillators which are located in other areas in the brain and in peripheral tissues [5].

Circadian rhythms in behavior, physiology, and metabolism are essential to sustain adequate organism homeostasis. Indeed, misalignment of the circadian clock can lead to severe disorders such as obesity, type II diabetes, or metabolic syndrome [7]. The control that the circadian clock exerts on 
cellular metabolism is complex and multilayered, yet numerous underlying molecular mechanisms are being unraveled. Intriguingly, a number of rate-limiting enzymes controlling the pace of critical metabolic pathways are under circadian control of expression or activity [8]. This regulation is exerted by the circadian clock, a well-coordinated transcription-translation feedback system that orchestrates and integrates gene expression, protein stability, and metabolite production to keep correct time. The core components of the circadian clock are transcription factors which impose rhythmicity to a significant portion of the transcriptome in a tissue or cell-specific manner [9]. Transcriptional rhythms are accompanied by rhythmic chromatin transitions supported by a highly plastic and dynamic circadian epigenome [10]. Here, we summarize findings showing how the circadian machinery shapes the chromatin landscape and the active role of specific metabolites in regulating these events. The coordination of circadian gene expression in specific nuclear territories through the physical association of genes in circadian interactomes is also reviewed.

\section{The Circadian Molecular Clock in Mammals}

Molecular clocks are present in almost all cells in the organism, and they share a molecular architecture with the ability to generate and sustain circadian rhythms on transcription of clock-controlled genes (CCG) [11]. Two transcriptional activators (CLOCK and BMAL1) and two repressors (CRY and PER proteins) compose the core clock machinery [12-16]. CLOCK and BMAL1 proteins heterodimerize and are rhythmically recruited to E-box elements located in the promoter of CCGs to subsequently activate their expression. The circadian repressors per 1-3 and cry 1-2 are among the CCGs. PER and CRY proteins accumulate and heterodimerize in the cytoplasm to subsequently translocate into the nucleus. This process is tightly regulated by posttranslational modification events, mostly involving phosphorylation of PER and CRY proteins by a number of kinases such as CKIE or CKI $\delta$ [17]. In the nucleus, PER:CRY complexes repress CLOCK:BMAL1-mediated transcriptional activity on CCGs, thereby generating a negative feedback loop. The stability of the PER:CRY complex is regulated by specific F-Box proteins including FBXL3, which target them for degradation by the proteasome $[18,19,20 \bullet \bullet, 21 \bullet \cdot]$. These events result into time-controlled clearance of repressors from the nucleus, priming for a new cycle of CLOCK:BMAL1-driven gene expression. This timely regulated molecular mechanism is key to the rhythmic expression of CCGs.

Among the CCGs, there are transcription factors that further extend the clock regulatory system by imposing rhythmicity to downstream genes. Among these factors there are several nuclear receptors, such as REV-ERB $\alpha /$ REV-ERB $\beta$ and the retinoid orphan receptors RORs ( ROR $\alpha,-\beta,-\gamma)$, which bind to ROR elements in the promoters and controls rhythmic expression on a subset of genes including bmall [22, 23]. Additional circadian transcription factors are exemplified by DBP, TEF, HLF, and E4BP4, whose transcriptional activities impose rhythmicity to genes involved in xenobiotic detoxification pathways in the liver [24].

\section{Dynamic Transitions on Chromatin}

Transcription factors exert their function by recruiting activators of the transcription machinery, some of which can modulate the epigenome by contributing to an open chromatin environment permissive for transcription. Conversely, transcriptional repressors promote chromatin condensation, therefore limiting accessibility and activity of the transcription machinery $[25,26]$. It is thereby required that specific chromatin modifiers in the cell nucleus cooperate with the clock to direct cyclic events which sustain circadian rhythms in transcription (Fig. 1). To this end, the circadian heterodimer CLOCK:BMAL1 interacts with histone acetyltransferases (HATs) and deacetylases (HDACs) to coordinate timed chromatin transitions in histone acetylation at CCGs promoters. Specifically, CLOCK:BMAL1 has been shown to interact with the HATs CBP (CREB binding protein), p300, and with the CBP-associated factor PCAF [27-30]. These molecular associations lead to cyclic oscillations in histone acetylation at CCG promoters, which in turn result in rhythmic chromatin transitions [11]. Moreover, the CLOCK protein itself is a HAT that rhythmically acetylates K9/K14 residues of histone $\mathrm{H} 3$ [31].

Histone acetylation by these various HATs is counterbalanced by specific HDACs. Notably, the transcriptional repressor complex SIN3-HDAC cooperates with PER proteins to elicit deacetylation of histones at perl gene promoter [32•]. Similarly, genomic recruitment of HDAC3 by REV-ERB $\alpha$ in mouse liver directs a circadian rhythm of histone deacetylation on a number of genes required for hepatic lipid homeostasis [33••]. Other HDACs important for circadian function are the one included in the class III of enzymes known as sirtuins. These enzymes are of particular interest, as their deacetylase activity depends on the metabolite nicotine adenine dinucleotide $\left(\mathrm{NAD}^{+}\right)$[34]. There are seven mammalian sirtuins (SIRT1-7), among which SIRT1, SIRT3, and SIRT6 are involved in circadian control of gene expression and metabolism $[35,36 \bullet \bullet, 37,38 \bullet \bullet$. Their characterization has provided the first molecular link between metabolism, epigenetics, and the circadian clock.

Specific histone methyltransferases (HMTs) have been related to clock function. For example, the mixed-lineage leukemia (MLL) family member MLL1 is critical to recruit the CLOCK:BMAL1 complex to chromatin and elicits rhythmic $\mathrm{H} 3 \mathrm{~K} 4$ tri-methylation at CCGs promoters, a mark that is 


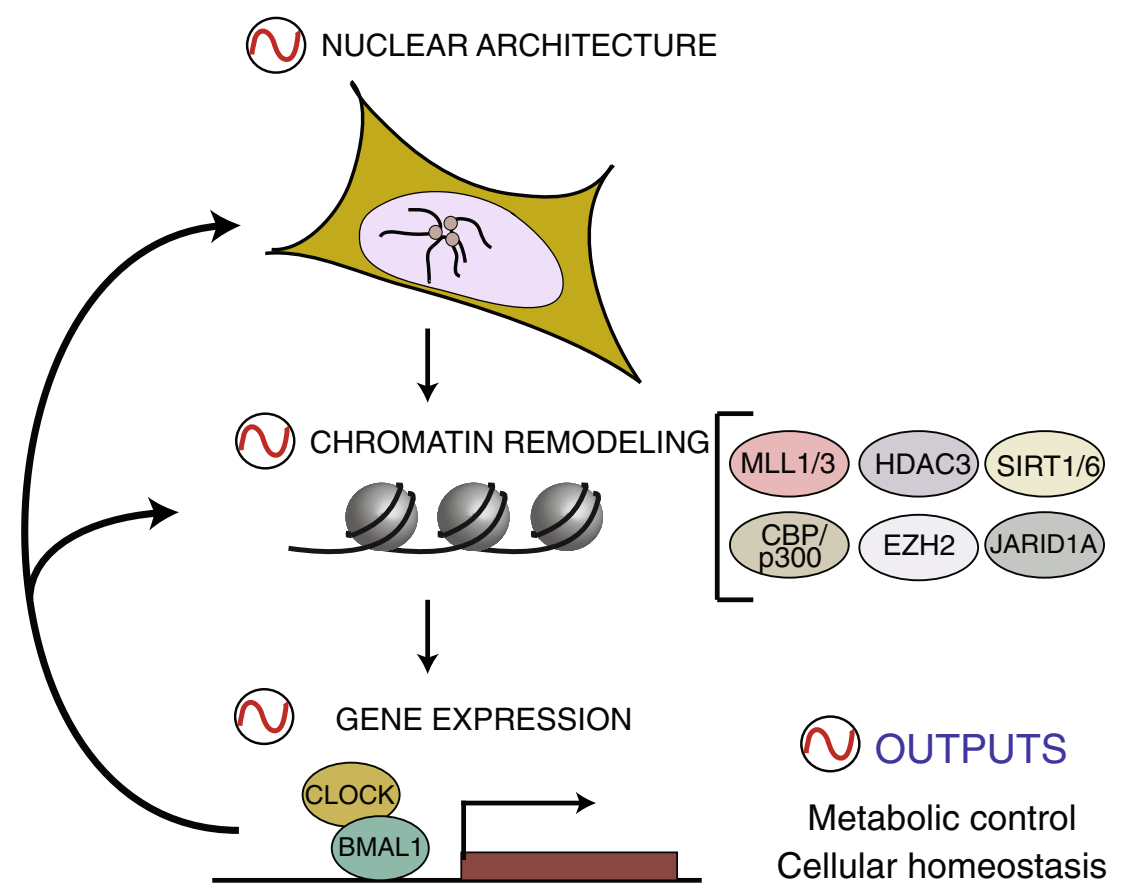

Fig. 1 Different regulatory layers delineate the circadian transcriptome. The circadian clock operates in the cell through interconnected mechanisms to regulate circadian gene expression. The tridimensional organization of circadian genes in circadian interactomes inside of the nucleus provides a framework for transcriptional regulation. Moreover, the clock coordinates with specific chromatin remodelers to generate

associated with transcriptional activation. Another member of the same family, MLL3, has also been shown to contribute to circadian control [39••, 40•]. Additionally, the circadian component PER2 interacts with the H3K9 methyltransferase Suv39h1 to mediate repression of the perl gene [41]. Moreover, the repressive mark H3K27me3 appears rhythmic at the level of per 1 and per 2 promoters, an event dependent on the HMT EZH2 [42].

Temporal and genome wide analyses of histone modifications and clock proteins DNA occupancy using high throughput chromatin immunoprecipitation (ChIP-seq) technologies have revealed many aspects of the circadian epigenome in the mouse liver $[11,43 \bullet \bullet, 44,45 \bullet]$. These studies confirm that CLOCK:BMAL1 complex recruitment to chromatin is temporally restricted to the light phase, between ZT4 and ZT8. Conversely, the circadian repressors CRY and PER appear antiphasic, during the dark period. The genome-wide pattern of circadian histone modifications is complex, and globally these are not restricted to a specific time of the day, but rather oscillate in order to sustain the distinct phase of each CCG $[43 \bullet \bullet, 45 \bullet]$. Overall, these events reveal the coordination of multiple epigenetic regulatory components to generate the adequate circadian output (Fig. 1).

Intriguingly, RNA-seq experiments in the mouse liver in combination with ChIP-seq analyses for temporal DNA occupancy profiles by RNA polymerase II indicate that not every chromatin transitions which support circadian transcription. The core clock proteins CLOCK and BMAL1 rhythmically bind to promoters of clock-controlled genes and drive circadian gene expression. Finally, the circadian transcriptional output imposes rhythmicity to metabolic pathways and specific signaling events to maintain cellular homeostasis

circadian transcript is subjected to rhythmic "de novo" transcription [43••, 44, 46•]. Instead, a significant portion of the circadian transcriptome is driven by clock-controlled posttranscriptional mechanisms such as rhythmic splicing events, mRNA stability and translation, or regulation by non-coding RNAs (ncRNAs) and microRNAs (miRNAs) [47, 48]. For example, cyclic alternative splicing occurs in the mouse liver, controlling the mRNA levels of circadian genes such as npas 2 [49]. Additionally, alterations in the m(6)A-RNA methylation machinery affects circadian period length and disturbs the pre$\mathrm{mRNA} /$ mature RNA subcellular distribution of the clock genes per2 and bmall [50••]. Moreover, a wide variety of ncRNAs are rhythmically expressed in the mouse liver including an antisense transcript on per 2 , which might have a regulatory function $[45 \cdot 51 \bullet \cdot$. Finally, genetic disruption of the microRNA pathway by mutating dicer affects the circadian transcriptome [52, 53•]. Indeed, specific miRNAs including miR-122, miR-139, and miR-219 appear to modulate the circadian clock in a tissue-specific manner $[54,55]$.

\section{A Genomic Interactome Driven by the Clock}

Specific posttranslational histone modifications have been associated to various levels of transcriptional control [25]. Studies on circadian chromatin remodeling provide time- 
specific linear maps for the localization of epigenetic marks to the DNA sequence and their implication with rhythmic transcription. However, understanding of genome function requires considering the spatial organization of interphase chromosomes inside of the nucleus [56]. Indeed, mammalian genomes are highly organized in topological domains with pervasive features across different cell types, tissues, or even species $[57,58]$. Functional territories include domains with active transcription towards the center of the nucleus and repressive domains mostly localized at the nuclear periphery and spatial segregation of epigenetic marks [59].

A recent study using chromosome conformation capture on chip (4C) technology demonstrates that mammalian circadian genes are organized in functional nuclear territories [60••]. In this study, mouse embryonic fibroblasts were used to decipher the genomic environment of the clock-controlled gene $d b p$ along the circadian cycle. The location of $d b p$ gene is consistently restricted to high gene dense nuclear domains, and specific interchromosomal contacts appear remarkably pervasive across the circadian cycle. A striking feature of the $d b p$ interactome is the spatial congregation of clock-related DNA-elements, including a significant enrichment in E-box elements $\left[60^{\bullet}\right]$. These data support a scenario where the various phases of the circadian program of gene expression occur in specific nuclear domains.

Interestingly, some specific $d b p$ genomic contacts are dynamic, and their interaction mirrors $d b p$ expression, revealing a circadian interactome [60• $]$. These interactions do not appear in bmall $^{-/-}$cells, indicating that the clock machinery is implicated in the $d b p$ genomic environment, possibly favoring adequate circadian transcriptional regulation. Notably, a subgroup of genes located within the $d b p$ circadian interactome is coordinately transcribed in a circadian fashion. This includes the H3K36 histone methyltransferase ash $1 l$ and the metabolic gene acy3. It is thereby conceivable that these genes are coregulated by sharing the transcription machinery within dedicated areas in the nucleus, possibly contributing to the assembly of specific circadian transcription factories $[60 \bullet$, 61-63]. Overall, these observations provide the first evidence of interplay between chromosome organization and the clock to regulate the circadian output in mammals. Additionally, the nuclear membrane has been demonstrated to regulate circadian rhythms, possibly by a direct interaction of the nuclear envelope component MAN1 with the promoter of the clock gene bmall, thereby activating its expression [64•]. Thus, circadian gene positioning inside of the nucleus appears as an important regulatory layer by which cells can organize the cyclic expression of a large number of genes (Fig. 1).

The extent of the influence that chromatin topological organization has on the mammalian circadian transcriptome deserves to be further studied. Notably, in the in the Cyanobacterium Synechococcus elongatus, the circadian clock regulates a chromosome compaction rhythm, and experimental manipulations of the chromosome topological state impact circadian gene expression in a predictable manner, indicating that genome folding defines its circadian output $[65 \cdot, 66]$.

\section{The NAD ${ }^{+}$-Sirtuins Pathway Connects Metabolism to Epigenetics}

Accumulating evidence demonstrates that the circadian epigenome and metabolism are intimately connected [3]. Studies from genetically modified mice lacking circadian components indicate that the clock controls a large portion of metabolic homeostasis and reveals important connections between disruption of circadian rhythms and disease [7]. For example, clock mutant mice are hyperphagic and obese and develop a severe metabolic syndrome [67]. Moreover, selective ablation of the circadian gene bmall within the pancreas leads to profound hyperglycemia and $\beta$-cell failure, leading to diabetes mellitus $[68 \bullet \cdot$. Additionally, mice with a liver-specific deletion of bmall are unable to maintain glucose homeostasis and exhibit hypoglycemia and defective expression of hepatic glucose regulatory genes [69]. It is thereby essential to decipher molecular connections between circadian rhythms and metabolism, as these could help design of pharmacological strategies towards the treatment of a number of metabolic diseases.

In this context, the $\mathrm{NAD}^{+}$-dependent class III family of deacetylases, named sirtuins, represents a revealing paradigm. There are seven mammalian sirtuins (SIRT1-7), of which SIRT1, SIRT3, and SIRT6 have been linked to the circadian clock [70]. SIRT1, the founding member of this family of enzymes, specifically deacetylates the circadian components BMAL1 and PER2 [35, 37], thereby impacting their activities. Also, SIRT1 modulates acetylation on H3K9/K14 at CCGs promoters $[37,71 \bullet]$. As a consequence, circadian gene expression is altered in the liver from mice with a hepatic-specific deletion of sirt1 [36••, 37]. Moreover, SIRT1 controls the expression of the bmall gene in the SCN, and influences circadian amplitude and period length [72•*]. Notably, the activity of SIRT1 is circadian, a function that has been linked to the cyclic availability of its cofactor $\operatorname{NAD}^{+}[73 \bullet \bullet, 74 \bullet \cdot]$. Interestingly, the rate-limiting step within the $\mathrm{NAD}^{+}$biosynthetic pathway is catalyzed by the enzyme NAMPT (nicotinamide phosphoribosyltransferase), and the gene nampt is clock controlled, through rhythmic binding of CLOCK:BMAL1 to E-boxes located on its promoter [73••, 74.0]. Thereby, $\mathrm{NAD}^{+}$biosynthesis appears circadian through the coupling of two transcriptional and enzymatic feedback loops. Rhythmic fluctuations in $\mathrm{NAD}^{+}$levels have been observed both in synchronized cells and tissues [73••, 74••].

Recent work reveals that the nuclear sirtuins SIRT1 and SIRT6 control circadian gene expression of differential groups of genes, defining distinctly partitioned classes of circadian 
genes $[36 \bullet \bullet, 70]$. Thereby, SIRT1 and SIRT6 operate through distinct mechanisms to delineate the circadian output. Although both of them interact with CLOCK:BMAL1, they differentially regulate the function of the heterodimer. Interestingly, SIRT6 controls CLOCK:BMAL 1 transactivation on promoters of genes involved in fatty acid biosynthesis, possibly by coordinating its function with specific transcription factors such as the sterol regulatory element-binding protein 1 (SREBP1) [36•*]. Moreover, the genomic partitioning by SIRT1 and SIRT6 results in parallel segregation of cellular metabolism. Specifically, SIRT6 dictates circadian metabolism of fatty acids, including triglycerides, membrane and signaling lipids, and medium- and longchain fatty acids $[36 \bullet \bullet$.

Additionally, the mitochondrial sirtuin SIRT3 shows cyclic deacetylase activity driven by circadian rhythms in $\mathrm{NAD}^{+}$ availability $[38 \bullet \bullet$. SIRT3 coordinates the mitochondrial acetylome in response to nutrient status to keep homeostasis. Particularly, SIRT3 regulates acetylation of proteins involved in several metabolic pathways such as fatty acid oxidation and tricarboxylic acid cycle $[75,76]$. Many of these acetylation events impact protein activity and appear circadian, suggesting that SIRT3 dictates rhythms in mitochondrial function $[38 \bullet$, 77]. Notably, rhythms in fatty acid oxidation are disrupted in $\mathrm{Bmall}^{-/-}$mice and can be restored by exogenous administration of $\mathrm{NAD}^{+}$, demonstrating that the clock contributes to the control of mitochondrial oxidative function [38••].

Through modulation of sirtuins, the cofactor $\mathrm{NAD}^{+}$is emerging as key regulator of metabolism and circadian rhythms. Interestingly, sirtuins control longevity in different species and are intimately related with aging process [34]. Also $\mathrm{NAD}^{+}$levels decay with aging $[78,79]$, and these changes are paralleled by mis-regulation in amplitude and phase of clock genes [80•, 81•]. In the central pacemaker, some of these age-dependent circadian defects are controlled by SIRT1 $\left[72 \bullet \cdot\right.$. Thereby, the $\mathrm{NAD}^{+}$- sirtuin pathway provides a link between metabolism, circadian rhythms, and longevity [82].

\section{Metabolites Talk to the Clock}

Oscillating metabolites are important for the maintenance of cellular rhythmicity, and $\mathrm{NAD}^{+}$is a revealing example. Present knowledge favors a scenario in which the clock appears to sense the cellular energy state and modify its transcriptional function to adapt. Indeed, certain metabolites have been shown to directly interact with components of the clock machinery. For example, it was proposed that CLOCK:BMAL1 DNA-binding affinities to E-boxes are affected by the redox states of NAD $(H)$ or NADP $(H)$, although "in vivo" evidence for these observations has been lacking [83]. Intriguingly, recent crystallographic studies suggest that the repressor complex CRY1:PER2 is destabilized by a disulfide bond between two cysteine residues in CRY1 [84••], which could act as a redox sensor modulating the complex. Moreover, the mammalian CRY2 protein presents a conserved FAD (flavin adenine dinucleotide) binding pocket, which is also targeted by the ubiquitin ligase protein FBXL3 $[18,19,85 \bullet \cdot]$. This interplay regulates CRY2 stability and impacts circadian period length $[85 \bullet \bullet, 86]$.

Additionally, posttranslational modifications of the clock machinery provide means to connect cellular metabolism and the oscillator. For example, the metabolite $O$-linked $\beta$ $\mathrm{N}$-acetylglucosamine (O-GlcNAc) can be linked to the circadian proteins CLOCK, BMAL1, and PER2 by the enzyme OGT (O-GlcNAc transferase), thereby modifying the turnover of the proteins $[87 \bullet, 88 \bullet$. Interestingly, O-GlcNAc is becoming increasingly recognized as a key signal transducer from glucose metabolism [89]. Furthermore, the nutrient sensor protein AMPK (AMP-activated protein kinase) was shown to directly phosphorylate the clock component CRY1, thereby destabilizing it. Notably, this particular posttranslational modification is sensitive to cellular glucose levels; thereby, AMPK functions as a signal transducer between glucose metabolism and the oscillator $[90 \bullet \bullet]$.

The extent of metabolic influence on clock function needs further investigation. The use of metabolomic approaches reveals that diet composition and nutritional cues have unforeseen effects on the circadian clock. For example, mice fed a high-fat diet become obese and diabetic, and remarkably they show a specific reorganization of metabolic pathways in the liver, leading to profound reprogramming of the hepatic clock $[91 \bullet \bullet]$. These changes are illustrated by advanced recruitment of CLOCK:BMAL1 heterodimer to promoters of CCGs and specific epigenetic events leading to deep alterations in the circadian transcriptome [91••]. Interestingly, these alterations are reversible by switching to normal diet, and also can be alleviated by time-restricted feeding [91••, 92•], highlighting the remarkable plasticity of the oscillator.

\section{Conclusion}

Accumulating data reveal the role of the circadian clock in maintaining cellular homeostasis. It is becoming increasingly evident that the oscillator senses and integrates information from metabolic pathways and modifies its function to adapt accordingly. Remarkably, the molecular clock, through the coordination of chromatin remodelers, modulates a vast epigenomic landscape, leading to plastic and dynamic chromatin transitions which delineate the specific circadian transcriptome. The integration of cellular signaling by the clock in the nucleus extends to regulation of circadian genes positioning, a mechanisms which might be critical to define the circadian output. The decoding of the language that links nutrients and metabolites to the circadian clock will be important to 
decipher how mis-regulation of circadian rhythms leads to chronic diseases such as obesity and diabetes.

\section{Compliance with Ethics Guidelines}

Conflict of Interest Lorena Aguilar-Arnal and Paolo Sassone-Corsi declare that they have no conflict of interest.

Human and Animal Rights and Informed Consent All animal studies performed by any of the authors were in accordance to the Institutional Animal Care and Use Committee (IACUC) guidelines at the University of California, Irvine.

\section{References}

Papers of particular interest, published recently, have been highlighted as:

- Of importance

•- Of major importance

1. Dunlap JC. Molecular bases for circadian clocks. Cell. 1999;96(2): 271-90.

2.• Edgar RS, Green EW, Zhao Y, van Ooijen G, Olmedo M, Qin X, et al. Peroxiredoxins are conserved markers of circadian rhythms. Nature. 2012;485(7399):459-64. The authors describe that non transcriptional rhythms in oxidation-reduction of peroxiredoxin proteins constitute a universal marker for circadian rhythms in all domains of life. These oscillations are present in a variety of model organisms including Bacteria, Archaea and Eucaryota, suggesting that sensing and responding to oxidative cycles could have driven the evolution of circadian rhythms.

3. Eckel-Mahan K, Sassone-Corsi P. Metabolism and the circadian clock converge. Physiol Rev. 2013;93(1):107-35.

4. LeGates TA, Fernandez DC, Hattar S. Light as a central modulator of circadian rhythms, sleep and affect. Nat Rev Neurosci. 2014;15(7):443-54.

5. Dibner C, Schibler U, Albrecht U. The mammalian circadian timing system: organization and coordination of central and peripheral clocks. Annu Rev Physiol. 2010;72:517-49.

6. Orozco-Solis R, Sassone-Corsi P. Epigenetic control and the circadian clock: linking metabolism to neuronal responses. Neuroscience. 2014;264:76-87.

7. Maury E, Ramsey KM, Bass J. Circadian rhythms and metabolic syndrome: from experimental genetics to human disease. Circ Res. 2010;106(3):447-62.

8. Sahar S, Sassone-Corsi P. Regulation of metabolism: the circadian clock dictates the time. Trends Endocrinol Metab. 2012;23(1):1-8.

9. Doherty CJ, Kay SA. Circadian control of global gene expression patterns. Annu Rev Genet. 2010;44:419-44.

10. Aguilar-Arnal L, Sassone-Corsi P. The circadian epigenome: how metabolism talks to chromatin remodeling. Curr Opin Cell Biol. 2013;25(2):170-6.

11. Partch CL, Green CB, Takahashi JS. Molecular architecture of the mammalian circadian clock. Trends Cell Biol. 2014;24(2):90-9.

12. Bunger MK, Wilsbacher LD, Moran SM, Clendenin C, Radcliffe LA, Hogenesch JB, et al. MOP3 is an essential component of the master circadian pacemaker in mammals. Cell. 2000;103(7):100917.

13. Gekakis N, Staknis D, Nguyen HB, Davis FC, Wilsbacher LD, King DP, et al. Role of the CLOCK protein in the mammalian circadian mechanism. Science. 1998;280(5369):1564-9.
14. Kume K, Zylka MJ, Sriram S, Shearman LP, Weaver DR, Jin X, et al. MCRY1 and mCRY2 are essential components of the negative limb of the circadian clock feedback loop. Cell. 1999;98(2):193205.

15. Vitaterna MH, King DP, Chang AM, Kornhauser JM, Lowrey PL, McDonald JD, et al. Mutagenesis and mapping of a mouse gene, clock, essential for circadian behavior. Science. 1994;264(5159): 719-25.

16. Zheng B, Albrecht U, Kaasik K, Sage M, Lu W, Vaishnav S, et al. Nonredundant roles of the mper 1 and mper 2 genes in the mammalian circadian clock. Cell. 2001;105(5):683-94.

17. Lee H, Chen R, Lee Y, Yoo S, Lee C. Essential roles of CKIdelta and CKIepsilon in the mammalian circadian clock. Proc Natl Acad Sci U S A. 2009;106(50):21359-64.

18. Busino L, Bassermann F, Maiolica A, Lee C, Nolan PM, Godinho SI, et al. SCFFBXL3 controls the oscillation of the circadian clock by directing the degradation of cryptochrome proteins. Science. 2007;316(5826):900-4

19. Siepka SM, Yoo SH, Park J, Song W, Kumar V, Hu Y, et al. Circadian mutant overtime reveals f-box protein FBXL3 regulation of cryptochrome and period gene expression. Cell. 2007;129(5): 1011-23.

20.• Hirano A, Yumimoto K, Tsunematsu R, Matsumoto M, Oyama M, Kozuka-Hata H, et al. FBXL21 regulates oscillation of the circadian clock through ubiquitination and stabilization of cryptochromes. Cell. 2013;152(5):1106-18. These papers describe that FBXL21, an F-box-type E3 ubiquitin ligase, targets CRY proteins, thereby protecting them from FBXL3 mediated degradation. Hence, a competition mechanism between FBXL21 and FBXL3 regulates CRY stability and circadian period length.

21.• Yoo SH, Mohawk JA, Siepka SM, Shan Y, Huh SK, Hong HK, et al. Competing E3 ubiquitin ligases govern circadian periodicity by degradation of CRY in nucleus and cytoplasm. Cell. 2013;152(5): 1091-105. These papers describe that FBXL21, an F-box-type E3 ubiquitin ligase, targets CRY proteins, thereby protecting them from FBXL3 mediated degradation. Hence, a competition mechanism between FBXL21 and FBXL3 regulates CRY stability and circadian period length.

22. Preitner N, Damiola F, Lopez-Molina L, Zakany J, Duboule D, Albrecht U, et al. The orphan nuclear receptor REV-ERBalpha controls circadian transcription within the positive limb of the mammalian circadian oscillator. Cell. 2002;110(2):251-60.

23. Sato TK, Panda S, Miraglia LJ, Reyes TM, Rudic RD, McNamara $\mathrm{P}$, et al. A functional genomics strategy reveals RORa as a component of the mammalian circadian clock. Neuron. 2004;43(4):52737.

24. Gachon F, Olela FF, Schaad O, Descombes P, Schibler U. The circadian par-domain basic leucine zipper transcription factors DBP, TEF, and HLF modulate basal and inducible xenobiotic detoxification. Cell Metab. 2006;4(1):25-36.

25. Borrelli E, Nestler EJ, Allis CD, Sassone-Corsi P. Decoding the epigenetic language of neuronal plasticity. Neuron. 2008;60(6): 961-74.

26. Li B, Carey M, Workman JL. The role of chromatin during transcription. Cell. 2007;128(4):707-19.

27. Curtis AM, Seo SB, Westgate EJ, Rudic RD, Smyth EM, Chakravarti D, et al. Histone acetyltransferase-dependent chromatin remodeling and the vascular clock. J Biol Chem. 2004;279(8): 7091-7.

28. Etchegaray JP, Lee C, Wade PA, Reppert SM. Rhythmic histone acetylation underlies transcription in the mammalian circadian clock. Nature. 2003;421(6919):177-82.

29. Lee Y, Lee J, Kwon I, Nakajima Y, Ohmiya Y, Son GH, et al. Coactivation of the CLOCK-BMAL1 complex by CBP mediates resetting of the circadian clock. J Cell Sci. 2010;123(Pt 20):354757. 
30. Takahata S, Ozaki T, Mimura J, Kikuchi Y, Sogawa K, FujiiKuriyama Y. Transactivation mechanisms of mouse clock transcription factors, mCLOCK and mARNT3. Genes Cells. 2000;5(9):739-47.

31. Doi M, Hirayama J, Sassone-Corsi P. Circadian regulator CLOCK is a histone acetyltransferase. Cell. 2006;125(3):497-508.

32. Duong HA, Robles MS, Knutti D, Weitz CJ. A molecular mechanism for circadian clock negative feedback. Science. 2011;332(6036):1436-9. Using mass spectrometry techniques, the authors uncover constituent proteins of PER complexes. Novel components include several RNA binding proteins such as PSF (polypyrimidine tract-binding protein-associated splicing factor). Notably, PSF recruits the corepressor SIN3A within PER complexes, which serves as scafold for certain HDACs.

33.• Feng D, Liu T, Sun Z, Bugge A, Mullican SE, Alenghat T, et al. A circadian rhythm orchestrated by histone deacetylase 3 controls hepatic lipid metabolism. Science. 2011;331(6022):1315-9. The circadian nuclear receptor $R E V$-ERB $\alpha$ rythmically recruits hepatic HDAC3 to promoters of genes regulating lipid metabolism. This mechanism directs a circadian rhythm of histone acetylation and gene expession essential for adequate hepatic liver homestasis.

34. Imai S, Guarente L. NAD ${ }^{+}$and sirtuins in aging and disease. Trends Cell Biol. 2014;24(8):464-71.

35. Asher G, Gatfield D, Stratmann M, Reinke H, Dibner C, Kreppel F, et al. SIRT1 regulates circadian clock gene expression through PER2 deacetylation. Cell. 2008;134(2):317-28.

36.• Masri S, Rigor P, Cervantes M, Ceglia N, Sebastian C, Cuiying X, Roqueta-Rivera M, Deng C, Orsborne T, Mostoslavsky R, Baldi P et al. Partitioning circadian transcription by SIRT6 leads to segregated control of cellular metabolism. Cell. 2014. Using transcriptomics and metabolomics in mouse liver, the authors establish contribution for the $\mathrm{NAD}^{+}$dependent deacetylase SIRT6 in regulating circadian gene expression of a specific group of circadian genes. This regulation extends to metabolic control.

37. Nakahata Y, Kaluzova M, Grimaldi B, Sahar S, Hirayama J, Chen $\mathrm{D}$, et al. The $\mathrm{NAD}^{+}$-dependent deacetylase SIRT1 modulates clockmediated chromatin remodeling and circadian control. Cell. 2008;134(2):329-40.

38. • Peek CB, Affinati AH, Ramsey KM, Kuo HY, Yu W, Sena LA, et al. Circadian clock $\mathrm{NAD}^{+}$cycle drives mitochondrial oxidative metabolism in mice. Science. 2013;342(6158):1243417. In this work, the mitochondrial located, $\mathrm{NAD}^{+}$dependent deacetylase SIRT3 is described to present circadian rhtyhms in deacetylase activity directed by rhythmic availbility of its cofactor $N A D^{+}$. These events are clock controlled. As a consequence, mitochondrial oxidative metabolism follows a circadian pattern of activity.

39.• Katada S, Sassone-Corsi P. The histone methyltransferase MLL1 permits the oscillation of circadian gene expression. Nat Struct Mol Biol. 2010;17(12):1414-21. The authors describe that H3K4me3 epigenetic mark is rhythmic at promoters of clock controlled genes. Moreover, these rhythms are dictated by a molecular interplay between CLOCK:BMAL1 and the histone methyltransferase MLL1.

40. Valekunja UK, Edgar RS, Oklejewicz M, van der Horst GT, O’Neill JS, Tamanini F, et al. Histone methyltransferase MLL3 contributes to genome-scale circadian transcription. Proc Natl Acad Sci U S A. 2013;110(4):1554-9. Using a combination of high throughput and biochemistry techniques, the authors present a role for the histone methyltransferase MLL3 in circadian transcription.

41. Duong HA, Weitz CJ. Temporal orchestration of repressive chromatin modifiers by circadian clock period complexes. Nat Struct Mol Biol. 2014;21(2):126-32.

42. Etchegaray JP, Yang X, DeBruyne JP, Peters AH, Weaver DR, Jenuwein $\mathrm{T}$, et al. The polycomb group protein EZH2 is required for mammalian circadian clock function. J Biol Chem. 2006;281(30):21209-15.
43.• Koike N, Yoo SH, Huang HC, Kumar V, Lee C, Kim TK, et al. Transcriptional architecture and chromatin landscape of the core circadian clock in mammals. Science. 2012;338(6105):349-54. Using ChIP-seq on mouse liver, the authors present a circadian time specific genomic map of many transcriptional components of the mammalian clock machinery. RNA polymerase II recruitment is similarly mapped, and these data are correlated with time-specific RNA-seq to uncover circadian transcription dynamics.

44. Le Martelot G, Canella D, Symul L, Migliavacca E, Gilardi F, Liechti R, et al. Genome-wide RNA polymerase II profiles and RNA accumulation reveal kinetics of transcription and associated epigenetic changes during diurnal cycles. PLoS Biol. 2012;10(11): e1001442.

45. Vollmers C, Schmitz RJ, Nathanson J, Yeo G, Ecker JR, Panda S. Circadian oscillations of protein-coding and regulatory RNAs in a highly dynamic mammalian liver epigenome. Cell Metab. 2012;16(6):833-45. As in ref. 43, the authors describe the genome wide recruitment of multiple chromatin-associated clock components.

46. Menet JS, Rodriguez J, Abruzzi KC, Rosbash M. Nascent-seq reveals novel features of mouse circadian transcriptional regulation. Elife. 2012;1:e0011. Using genome-wide sequencing of nascent $R N A$, this research describes rhythms in de novo transcription. Unexpectedly, only a small portion of the mouse hepatic circadian transcriptome is driven by transcriptional rhtyhms.

47. Beckwith EJ, Yanovsky MJ. Circadian regulation of gene expression: at the crossroads of transcriptional and post-transcriptional regulatory networks. Curr Opin Genet Dev. 2014;27:35-42.

48. Kojima S, Shingle DL, Green CB. Post-transcriptional control of circadian rhythms. J Cell Sci. 2011;124(Pt 3):311-20.

49. McGlincy NJ, Valomon A, Chesham JE, Maywood ES, Hastings MH, Ule J. Regulation of alternative splicing by the circadian clock and food related cues. Genome Biol. 2012;13(6):R54.

$50 . \bullet$ Fustin JM, Doi M, Yamaguchi Y, Hida H, Nishimura S, Yoshida M, et al. RNA-methylation-dependent RNA processing controls the speed of the circadian clock. Cell. 2013;155(4):793-806. This paper shows that inhibition of transmethylation reactions elongates circadian period. This effect is at least in part mediated by disregulation of the $m^{6} A-R N A$ methylation machinery. Importantly, $m^{6} A$ RNA methylation controls nucleocytoplasmic distribution of transcripts from Per2 and Bmall genes.

51.• Xue Z, Ye Q, Anson SR, Yang J, Xiao G, Kowbel D, Glass NL, Crosthwaite SK, Liu Y. Transcriptional interference by antisense RNA is required for circadian clock function. Nature. 2014. The authors describe that a long noncoding antisense RNA derived from a core clock gene in Neurospora is required for circadian transcription.

52. Chen R, D'Alessandro M, Lee C. MiRNAs are required for generating a time delay critical for the circadian oscillator. Curr Biol. 2013;23(20):1959-68.

53. Du NH, Arpat AB, De Matos M, Gatfield D. MicroRNAs shape circadian hepatic gene expression on a transcriptome-wide scale. Elife. 2014;3:e02510. In this paper, a circadian profiling of $m R N A s$ and pre-mRNAs from Dicer-deficient mouse livers is compared to wild type, showing that miRNA pathway is important to the circadian hepatic transcriptome.

54. Cheng HY, Papp JW, Varlamova O, Dziema H, Russell B, Curfman JP, et al. MicroRNA modulation of circadian-clock period and entrainment. Neuron. 2007;54(5):813-29.

55. Gatfield D, Le Martelot G, Vejnar CE, Gerlach D, Schaad O, Fleury-Olela F, et al. Integration of microRNA mir-122 in hepatic circadian gene expression. Genes Dev. 2009;23(11):1313-26.

56. Bickmore WA. The spatial organization of the human genome. Annu Rev Genomics Hum Genet. 2013;14:67-84.

57. Dixon JR, Selvaraj S, Yue F, Kim A, Li Y, Shen Y, et al. Topological domains in mammalian genomes identified by analysis of chromatin interactions. Nature. 2012;485(7398):376-80. 
58. Ho JW, Jung YL, Liu T, Alver BH, Lee S, Ikegami K, et al. Comparative analysis of metazoan chromatin organization. Nature. 2014;512(7515):449-52.

59. Ciabrelli F, Cavalli G. Chromatin driven behavior of topologically associating domains. J Mol Biol. 2014. doi:10.1016/j.jmb.2014.09. 013.

60.• Aguilar-Arnal L, Hakim O, Patel VR, Baldi P, Hager GL, SassoneCorsi P. Cycles in spatial and temporal chromosomal organization driven by the circadian clock. Nat Struct Mol Biol. 2013;20(10): 1206-13. Using a combination of chromosome-conformation capture on chip (4C) and transcriptomics technologies with a high temporal resolution, the authors define the contribution of nuclear architecture to circadian gene expression. The association of genes in circadian interactomes is also described.

61. Cisse II, Izeddin I, Causse SZ, Boudarene L, Senecal A, Muresan L, et al. Real-time dynamics of RNA polymerase II clustering in live human cells. Science. 2013;341(6146):664-7.

62. Schoenfelder S, Sexton T, Chakalova L, Cope NF, Horton A, Andrews S, et al. Preferential associations between co-regulated genes reveal a transcriptional interactome in erythroid cells. Nat Genet. 2010;42(1):53-61.

63. Aguilar-Arnal L, Sassone-Corsi P. Chromatin landscape and circadian dynamics: spatial and temporal organization of clock transcription. Proc Natl Acad Sci USA. 2014. doi:10.1073/pnas. 1411264111

64. Lin ST, Zhang L, Lin X, Zhang LC, Garcia VE, Tsai CW, et al. Nuclear envelope protein MAN1 regulates clock through BMAL1. Elife. 2014;3:e02981. This paper describes that the core clock gene Bmall is physically recruited to the nuclear periphery by the membrane protein MAN1, suggesting a role for circadian gene positioning in transcriptional control.

65. V Vijayan V, Zuzow R, O’Shea EK. Oscillations in supercoiling drive circadian gene expression in Cyanobacteria. Proc Natl Acad Sci U S A. 2009;106(52):22564-8. The authors show that the topological status of the chromosome in the Cyanobacterium Synechococcus elongatus correlates with circadian gene expression.

66. Woelfle MA, Xu Y, Qin X, Johnson CH. Circadian rhythms of superhelical status of DNA in Cyanobacteria. Proc Natl Acad Sci U S A. 2007;104(47):18819-24.

67. Turek FW, Joshu C, Kohsaka A, Lin E, Ivanova G, McDearmon E, et al. Obesity and metabolic syndrome in circadian clock mutant mice. Science. 2005;308(5724):1043-5.

68.• Marcheva B, Ramsey KM, Buhr ED, Kobayashi Y, Su H, Ko CH, et al. Disruption of the clock components clock and bmall leads to hypoinsulinaemia and diabetes. Nature. 2010;466(7306):627-31. Using physiological studies in mice with genetic ablation of the circadian components clock and bmall, the authors provide a link between dysregulation of the pancreatic clock and the development of metabolic diseases such as diabetes.

69. Lamia KA, Storch KF, Weitz CJ. Physiological significance of a peripheral tissue circadian clock. Proc Natl Acad Sci U S A. 2008;105(39):15172-7.

70. Masri S, Sassone-Corsi P. Sirtuins and the circadian clock: bridging chromatin and metabolism. Sci Signal. 2014;7(342):re6.

71. Bellet MM, Nakahata Y, Boudjelal M, Watts E, Mossakowska DE, Edwards KA, et al. Pharmacological modulation of circadian rhythms by synthetic activators of the deacetylase SIRT1. Proc Natl Acad Sci U S A. 2013;110(9):3333-8. The authors reveal that using specific SIRT1 activator compounds, circadian rhythms in gene expression can be modulated both "in vivo" and "in vitro".

72.• Chang HC, Guarente L. SIRT1 mediates central circadian control in the SCN by a mechanism that decays with aging. Cell. 2013;153(7): 1448-60. This paper describes that the $\mathrm{NAD}^{+}$dependent deacetylase SIRT1 in the brain governs central circadian control by activating transcription of Clock and Bmall genes. Importantly,
SIRT1 levels in the SCN decrease with aging, impacting circadian gene expression and giving rise to a longer circadian period.

73.• Nakahata Y, Sahar S, Astarita G, Kaluzova M, Sassone-Corsi P. Circadian control of the $\mathrm{NAD}^{+}$salvage pathway by CLOCKSIRT1. Science. 2009;324(5927):654-7. These papers describe that the levels of the $N A D^{+}$salvage pathway rate limiting enzyme NAMPT are controlled by the clock through rhythmic binding of CLOCK:BMAL1 heterodimers to E-boxes located at the Nampt gene promoter. These mechanisms drive rhythmic biosinthesis and availability of the metabolite $N A D^{+}$.

74.• Ramsey KM, Yoshino J, Brace CS, Abrassart D, Kobayashi Y, Marcheva B, et al. Circadian clock feedback cycle through NAMPT-mediated NAD ${ }^{+}$biosynthesis. Science. 2009;324(5927): 651-4. These papers describe that the levels of the NAD salvage pathway rate limiting enzyme NAMPT are controlled by the clock through rhythmic binding of CLOCK:BMAL1 heterodimers to Eboxes located at the Nampt gene promoter. These mechanisms drive rhythmic biosynthesis and availability of the metabolite $\mathrm{NAD}^{+}$.

75. Hebert AS, Dittenhafer-Reed KE, Yu W, Bailey DJ, Selen ES, Boersma MD, et al. Calorie restriction and SIRT3 trigger global reprogramming of the mitochondrial protein acetylome. Mol Cell. 2013;49(1):186-99.

76. Rardin MJ, Newman JC, Held JM, Cusack MP, Sorensen DJ, Li B, et al. Label-free quantitative proteomics of the lysine acetylome in mitochondria identifies substrates of SIRT3 in metabolic pathways. Proc Natl Acad Sci U S A. 2013;110(16):6601-6.

77. Masri S, Patel VR, Eckel-Mahan KL, Peleg S, Forne I, Ladurner $\mathrm{AG}$, et al. Circadian acetylome reveals regulation of mitochondrial metabolic pathways. Proc Natl Acad Sci U S A. 2013;110(9):3339_ 44.

78. Gomes AP, Price NL, Ling AJ, Moslehi JJ, Montgomery MK, Rajman L, et al. Declining NAD $(+)$ induces a pseudohypoxic state disrupting nuclear-mitochondrial communication during aging. Cell. 2013;155(7):1624-38.

79. Mouchiroud L, Houtkooper RH, Moullan N, Katsyuba E, Ryu D, Canto $\mathrm{C}$, et al. The $\mathrm{NAD}(+) /$ sirtuin pathway modulates longevity through activation of mitochondrial UPR and FOXO signaling. Cell. 2013;154(2):430-41.

80. Libert S, Bonkowski MS, Pointer K, Pletcher SD, Guarente L. Deviation of innate circadian period from $24 \mathrm{~h}$ reduces longevity in mice. Aging Cell. 2012;11(5):794-800. The authors describe that mice with intrinsic period length close to 24 hours present increased life spans than those with shorter or longer innate circadian periods.

81. Wyse CA, Coogan AN. Impact of aging on diurnal expression patterns of clock and bmall in the mouse brain. Brain Res. 2010;1337:21-31. This paper shows that the expression patterns of CLOCK and BMAL1 become altered during aging in different areas of the brain.

82. Orozco-Solis R, Sassone-Corsi P. Circadian clock: linking epigenetics to aging. Curr Opin Genet Dev. 2014;26C:6672.

83. Rutter J, Reick M, Wu LC, McKnight SL. Regulation of CLOCK and NPAS2 DNA binding by the redox state of NAD cofactors. Science. 2001;293(5529):510-4.

84. • Schmalen I, Reischl S, Wallach T, Klemz R, Grudziecki A, Prabu $\mathrm{JR}$, et al. Interaction of circadian clock proteins CRY1 and PER2 is modulated by zinc binding and disulfide bond formation. Cell. 2014;157(5):1203-15. The authors decipher the crystal structure of a complex between CRY1 and PER2. Unexpectedly, a zinc ion and a disulfide bond formation coordinates the complex formation, providing a link with cellular redox state.

85.• Xing W, Busino L, Hinds TR, Marionni ST, Saifee NH, Bush MF, et al. Scf(FBXL3) ubiquitin ligase targets cryptochromes at their cofactor pocket. Nature. 2013;496(7443):64-8. This paper provides crystal structures of CRY2:FBXL3 complex. Interestingly, 
mammalian CRY2 retains binding affinity for the metabolite FAD, which competes for the binding pocket with FBXL3, hence modulating period length.

86. Nangle S, Xing W, Zheng N. Crystal structure of mammalian cryptochrome in complex with a small molecule competitor of its ubiquitin ligase. Cell Res. 2013;23(12):1417-9.

87. Kaasik K, Kivimae S, Allen JJ, Chalkley RJ, Huang Y, Baer K, et al. Glucose sensor O-glcnacylation coordinates with phosphorylation to regulate circadian clock. Cell Metab. 2013;17(2):291302. These papers show that O-GlcNAcylation of clock proteins provides means to adjust circadian rhythmicity and connects the clock to glucose signaling.

88. Li MD, Ruan HB, Hughes ME, Lee JS, Singh JP, Jones SP, et al. OGlcNac signaling entrains the circadian clock by inhibiting BMAL1/CLOCK ubiquitination. Cell Metab. 2013;17(2):303-10. These papers show that O-GlcNAcylation of clock proteins provides means to adjust circadian rhythmicity and connects the clock to glucose signaling.

89. Ruan HB, Singh JP, Li MD, Wu J, Yang X. Cracking the O-GlcNac code in metabolism. Trends Endocrinol Metab. 2013;24(6):301-9.
90.• Lamia KA, Sachdeva UM, DiTacchio L, Williams EC, Alvarez JG, Egan DF, et al. AMPK regulates the circadian clock by cryptochrome phosphorylation and degradation. Science. 2009;326(5951):437-40. The authors report that the nutrient sensor AMPK directly phosphorylates CRY1 targeting it for degradation. Interestingly, AMPK activity is rhythmic and provides a connection between nutrient signaling and circadian clocks.

91.• Eckel-Mahan KL, Patel VR, de Mateo S, Orozco-Solis R, Ceglia NJ, Sahar S, et al. Reprogramming of the circadian clock by nutritional challenge. Cell. 2013;155(7):1464-78. Using comparative analyses from metabolome and transcriptome in livers from mice fed either normal chow or a high fat diet, the authors conclude that nutritional cues impact circadian function by a mechanisms involving reprogramming of CLOCK:BMAL1-mediated transcription.

92. Hatori M, Vollmers C, Zarrinpar A, DiTacchio L, Bushong EA, Gill $\mathrm{S}$, et al. Time-restricted feeding without reducing caloric intake prevents metabolic diseases in mice fed a high-fat diet. Cell Metab. 2012;15(6):848-60. This work describes that restricted time of feeding without reducing calorie intake improves clock function. 